\title{
Correlation of Liquid Volume in Stomach to Electrical Transfer Impedance Measurements Using FIM
}

\author{
Masum Iquebal ${ }^{1}$ and K Siddique-e Rabbani \\ Department of Biomedical Physics \& Technology, University of Dhaka, Bangladesh \\ ${ }^{1}$ Presently at: Department of Physics, Dhaka college, Dhaka. \\ email: masum3k@yahoo.com, rabbani@univdhaka.edu \\ Received: 12 October 2013, Accepted: 15 December, 2013
}

\begin{abstract}
:
The present work is directed to the measurement of the absolute volume of secreted acid in the stomach using 6-electrode FIM. Stomach acid is important because it has diagnostic applications in diarrhoeal diseases and in anaemia. Through a simplified resistance model this work has predicted that the variation in $\left(Z_{\mathrm{e}}-Z_{\mathrm{s}}\right) / \mathrm{Z}_{\mathrm{e}}$ with volume of liquid in stomach should be much greater than that of $Z_{s} / Z_{e}$. The simple resistance model has also predicted that the normalised change in measured transfer impedance $\left(Z_{\mathrm{e}}-Z_{\mathrm{s}}\right) / \mathrm{Z}_{\mathrm{e}}$ will be proportional to the volume of liquid in the stomach provided the resistivity of the liquid and the depth of stomach from the frontal skin surface is constant. Both these have been verified through real life measurement on two human subjects. This means that the measured transfer impedance change can indeed be related to the volume of saline in stomach. Together with depth information obtained using anatomical markers, it may be possible to obtain the absolute volume using FIM non-invasively.
\end{abstract}

\section{INTRODUCTION}

The present work was taken up with the aim of measuring the absolute volume of secreted acid inside a human stomach non-invasively using Focused Impedance Method (FIM), a new technique conceived and developed at Dhaka University (Rabbani et al 1999). A reduced amount of secreted acid indicates proneness to diarrhea (Blonski et al 2013). Therefore, measurement of acid volume in individuals would help in raising the awareness of the subjects so that persons with reduced acid secretion can take adequate precautions to avoid diarrhea and thus maintain a good health. It is also known that a person having less acid secretion has less iron absorption which may cause anaemia (Sarkar et al 2013). Therefore, a simple non-invasive measurement of absolute volume of secreted acid would be very useful for people, particularly in the Third world countries like Bangladesh where diarrhea and anaemia are still prevalent.

At present there is no non-invasive technique to determine the volume of acid in stomach. The existing technique uses intubation of the stomach through the nostrils and then drawing out the acid using a syringe, which is unpleasant and not acceptable for large scale screening of individuals. Previously our extended group was successful in determining relative volume of secreted gastric acid using Electrical Impedance Tomography (EIT) (Sarkar et al 1997), where acid secretion was stimulated using appropriate drugs. EIT is a sophisticated technique using 16 or more electrodes around the abdomen. FIM, on the other hand, is a simple technique employing 8,6 or 4 electrodes in its three versions (Islam et al 2009) which can be applied from the front of the abdomen, and would be much less expensive. However, the sensitive zone of FIM includes organs and tissues other than the stomach, therefore, techniques need to be developed to isolate the measurement in the stomach region from that outside the stomach. 
Since FIM is not yet widely known, a brief introduction to 6-electrode FIM (Rabbani et al 1999), which was used in the present work, is appropriate at this point. An alternating current of constant amplitude (called a constant current) is branched out into two circuit loops such that the two resulting outputs are the same in amplitude and phase but are electrically isolated from each other. This is achieved through the use of two isolating transformers. These two current outputs are driven through two orthogonal pairs of electrodes shown as A-B and C-D respectively in Figure 1. These are to be called current electrode pairs in this paper. The resulting potential dropped across another pair of electrodes, U-V as shown, is measured using an appropriately designed circuitry. Through appropriate choice of phase of the two isolated current drive signals, the potentials developed across U-V due to both the orthogonal current drives can simply add up algebraically. Since the current amplitudes are constant, the potential is

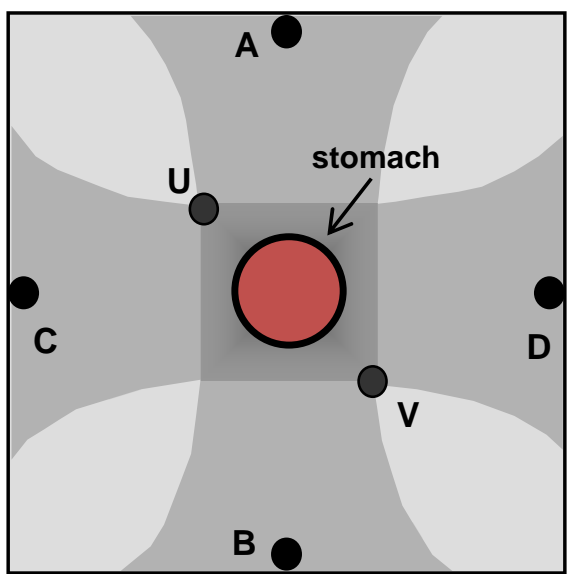

Fig. 1: Electrode positions in 6 electrode FIM directly proportional to the transfer impedance of the FIM system. The contribution of the central square region bounded by UV as the diagonal to the measured transfer impedance is very high compared to that of the regions outside. This led to the name, 'Focused Impedance, which was verified both through phantom measurements (Iquebal and Rabbani 2010, Rabbani et al 1999) and analytical means (Islam et al 2010).

FIM described above is basically a 2D technique. However, if the electrodes are placed on the surface of a 3D volume, due to 3D distribution of current in a volume conductor, the transfer impedance of regions within a certain depth of the electrode plane contributes to the measurement, although with reduced sensitivity. This, in fact is the rationale behind using surface electrodes placed on the abdomen to investigate the volume of secreted acid in the stomach.

The 3D effect of a target object has been studied in some detail by our group earlier using FIM (Iquebal and Rabbani 2010). Depth sensitivity of spherical conductors, insulators and of pieces of potato (cut to shape), each with different diameters, was measured. For all these objects, the sensitivity dropped sharply with depth initially, gradually leveling off to background with increasing depth. From this study, it was found that objects could be sensed down to a depth almost equal to the separation of the current electrodes.

Previously 6-electrode FIM has been applied successfully for the study of stomach emptying after a drink of saline (Rabbani et al 1999), which measured the relative change in FIM value with time before and after the drink. The transfer impedance decreased sharply immediately after the drink, which gradually increased back to the earlier value in about 20 to 25 minutes. This is expected to happen as the water is dispelled from the stomach into the duodenum and the small intestine. The results agreed very well to that obtained using region of interest analysis of EIT images (Rabbani et al 1999). However, in these experiments no attempts were made to measure the absolute volume of the liquid in the stomach, these only gave relative variation.

As mentioned before the measured transfer impedance on the abdomen will include both the stomach and the tissues around it that falls within the purview of the electrode system, while the interest is to measure the transfer impedance of the stomach region only. In fact the sensitivity of tissues lying 
above the stomach, nearer to the electrode plane, will have more sensitivity. Therefore, techniques need to be developed to isolate the effect of the target object from the surrounding tissues and organs. The contribution of tissues other than the target object may be overcome in certain situations where the transfer impedance of the target changes with time while that of its surroundings remain unchanged. In case of the stomach acid secretion, this will change with time while the other tissues will remain the same. Therefore, looking for changes in the transfer impedance with time may help extract the contribution of the acid variation from the contributions of the non-changing organs in the neighbouring regions. Acid secretion is a slow process, therefore, in the present work drinks of saline of different known volumes were used to find out if it is possible to measure the volume of the saline ingested using FIM through direct measurement on human subjects. In order to understand the method better, first a simple resistance model was developed to simulate the real life situation and then the predictions were compared with the measurements through different analyses. This, if successful, would also validate the proposed simple model.

\section{METHODS}

For the present work the 6-electrode version of FIM, as described above, was used. The measured electrical transfer impedance gets contribution from the whole volume that includes a target object of interest. The contribution of the target object to the measured transfer impedance depends on three factors, the size of the object given by its volume $\mathrm{V}$, the resistivity of the object $\rho$, and its depth $\mathrm{d}$. Thus the part of the measured transfer impedance arising out of the target object may be given as,

$$
\mathrm{Z}_{0}=\mathrm{Z}_{0}(\mathrm{~V}, \rho, \mathrm{d})
$$

This shows that relating $\mathrm{Z}_{0}$ to $\mathrm{V}$ is not straightforward and some assumptions and approximations are needed to achieve a practical solution. For the present work it is assumed that the resistivity $\rho$ of secreted acid for all individuals is constant. This may not be exactly true in all cases, but is reasonable to assume to get a clinically acceptable but approximate value of volume. Furthermore, if depth $d$ is found from prior knowledge of anatomy, then volume $\mathrm{V}$ of the acid in the stomach region may be determined from $\mathrm{Z}_{0}$.

However, the main challenge is to isolate $\mathrm{Z}_{0}$ from the measured transfer impedance $\mathrm{Z}$ which, as mentioned before, has contribution of $Z_{0}$ and the impedance of the surrounding region in a complex combination. In the present work a very simplistic impedance model is first developed to represent the FIM measurement situation from which an attempt is made to find out parameters that can help isolate $\mathrm{Z}_{0}$ from the total measurement. Then a study of FIM after a drink of saline is made on two subjects having similar age, gender, weight and height to find whether the predicted separation works out in practice. The two subjects, being very similar, are expected to give almost the same value of transfer impedance and the separated out part $\mathrm{Z}_{0}$ if the method developed is reasonably correct.

\subsection{The simplistic impedance model}

In the 6-electrode FIM current is driven through 2 orthogonal pairs of electrodes and the resulting potential difference is measured across a diagonal pair of electrodes, as mentioned before. The impedances between the electrodes are distributed in 3D in a complex way. In the simple model shown in Figure 2, two pairs of current electrodes in FIM are represented as a single pair I-I. The two potential electrodes are shown as $\mathrm{V}-\mathrm{V}$. The inside of the body that is sensitive to the impedance measurement (between electrodes $\mathrm{V}-\mathrm{V}$ ) has been considered in this model as a combination of series and parallel impedances as shown in the lightly shaded region where the red coloured region 
represents the stomach. The impedances shown represent lumped impedances arranged in this simple model, where $\mathrm{Z}_{22}$ represents that of the target object; in this case, the stomach. The measured voltage across $\mathrm{V}-\mathrm{V}$ allows measurement of the combined focused impedance $\mathrm{Z}$.

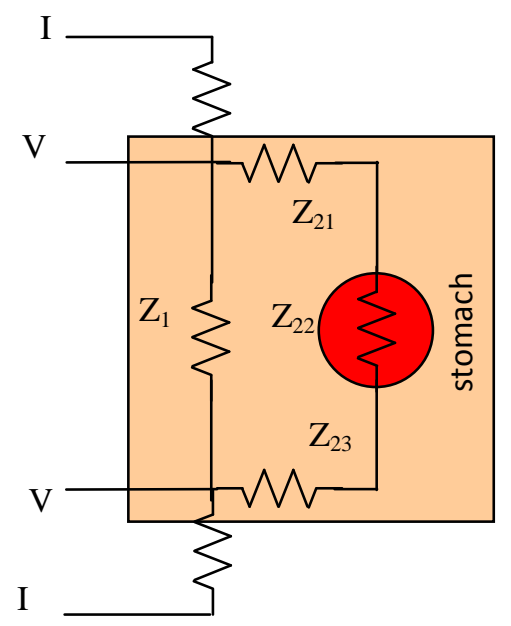

Figure-2. Impedance model for abdominal measurement of stomach using FIM

Let $\quad Z_{2}=Z_{21}+Z_{22}+Z_{23}$

Then, $\mathrm{Z}=\mathrm{Z}_{1}|| \mathrm{Z}_{2}=\mathrm{Z}_{1} \mathrm{Z}_{2} /\left(\mathrm{Z}_{1}+\mathrm{Z}_{2}\right)$

Since the changes in $Z_{22}$ will be small compared to the total value of $Z_{1}+Z_{2}$, the denominator in the right hand side of equation 2 may be assumed to be almost constant before and after the drink of saline. Using subscripts $\mathrm{e}$ and $\mathrm{s}$ to represent the situations with empty stomach and saline filled stomach respectively, $Z_{\mathrm{e}}$ and $\mathrm{Z}_{\mathrm{s}}$ may be expressed as, using equation 2 ,

$\mathrm{Z}_{\mathrm{e}}=\mathrm{Z}_{1} \mathrm{Z}_{2 \mathrm{e}} /\left(\mathrm{Z}_{1}+\mathrm{Z}_{2 \mathrm{e}}\right) \approx \mathrm{Z}_{1} \mathrm{Z}_{2 \mathrm{e}} /\left(\mathrm{Z}_{1}+\mathrm{Z}_{2}\right)$

$\mathrm{Z}_{\mathrm{s}}=\mathrm{Z}_{1} \mathrm{Z}_{2 \mathrm{~s}} /\left(\mathrm{Z}_{1}+\mathrm{Z}_{2 \mathrm{~s}}\right) \approx \mathrm{Z}_{1} \mathrm{Z}_{2 \mathrm{~s}} /\left(\mathrm{Z}_{1}+\mathrm{Z}_{2}\right)$

The difference, $\left(\mathrm{Z}_{\mathrm{e}}-\mathrm{Z}_{\mathrm{s}}\right) \approx\left(\mathrm{Z}_{2 \mathrm{e}}-\mathrm{Z}_{2 \mathrm{~s}}\right) \mathrm{Z}_{1} /\left(\mathrm{Z}_{1}+\mathrm{Z}_{2}\right)$

Since $Z_{21}$ and $Z_{23}$ remain unchanged, Eq. 1 gives,

$\mathrm{Z}_{2 \mathrm{e}}-\mathrm{Z}_{2 \mathrm{~s}}=\mathrm{Z}_{22 \mathrm{e}}-\mathrm{Z}_{22 \mathrm{~s}}$

Since $Z_{1} /\left(Z_{1}+Z_{2}\right)$ is constant in a set of measurements on a single subject with changes only in the stomach region,

$\left(\mathrm{Z}_{\mathrm{e}}-\mathrm{Z}_{\mathrm{s}}\right) \alpha\left(\mathrm{Z}_{22 \mathrm{e}}-\mathrm{Z}_{22 \mathrm{~s}}\right)$

This means that the difference of two abdominal measurements of FIM before and after a drink of saline is directly proportional to the impedance of the stomach region that contains the liquid taken.

Apart from the difference, a ratio parameter, $\mathrm{Z}_{\mathrm{s}} / \mathrm{Z}_{\mathrm{e}}$ was also studied. 
From equations 3 and 4 the ratio becomes,

$\mathrm{Z}_{\mathrm{s}} / \mathrm{Z}_{\mathrm{e}}=\mathrm{Z}_{2 \mathrm{~s}} / \mathrm{Z}_{2 \mathrm{e}}$

which in expanded form is (using eq. 1),

$\mathrm{Z}_{\mathrm{s}} / \mathrm{Z}_{\mathrm{e}}=\mathrm{Z}_{2 \mathrm{~s}} / \mathrm{Z}_{2 \mathrm{e}}=\left(\mathrm{k}+\mathrm{Z}_{22 \mathrm{~s}}\right) /\left(\mathrm{k}+\mathrm{Z}_{22 \mathrm{e}}\right)$

... (9)

Where $\mathrm{k}=\mathrm{Z}_{21}+\mathrm{Z}_{23}$

... (10)

From equations 7 and 9 , it can be seen that between the quantities $Z_{\mathrm{s}} / Z_{\mathrm{e}}$ and $\left(Z_{\mathrm{e}}-Z_{\mathrm{s}}\right)$, the former will vary less with the variation of the volume of liquid in the stomach as both the numerator and the denominator has added transfer impedances, $Z_{21}$ and $Z_{23}$ that remain unchanged.

It is planned to study the behaviours of both $Z_{s} / Z_{e}$ and $\left(Z_{\mathrm{e}}-Z_{\mathrm{s}}\right)$ in real life experiment with different amounts of

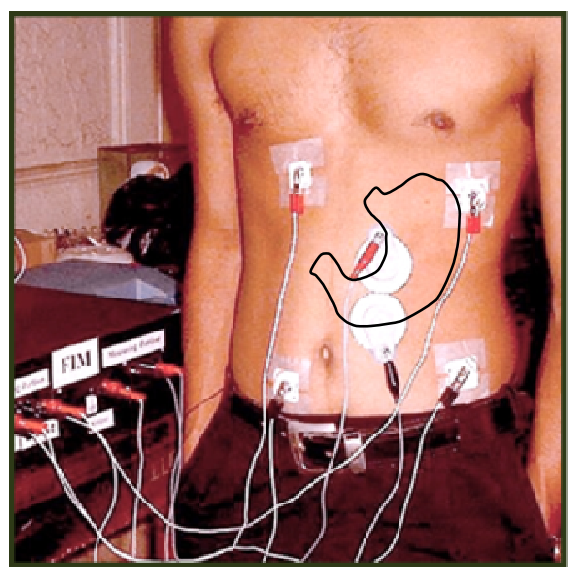

Figure-3. Electrode placement liquid in the stomach to see whether the simple model in Fig.2 is supported through the above equations.

The next question tried to answer in the present work was whether the change in the relative transfer impedance of the stomach region as measured using $\left(Z_{e}-Z_{s}\right)$ is related to the volume of the liquid in the stomach, or its area, or radius, assuming the liquid to take up a spherical space. This spherical assumption is of course grossly simplified, however, it is expected to give a clue in terms of the dependence of $\left(Z_{\mathrm{e}}-Z_{\mathrm{s}}\right)$.

For the above purposes real life experiments on two male human subjects were carried out. They had similar age and were of similar body structure, as given in Table 1.

Table 1: Physical parameters of the two male subjects

\begin{tabular}{|c|c|c|c|}
\hline & Age, yrs & Height, $\mathrm{m}$ & Weight, kg \\
\hline Subject-1 & 21 & 1.65 & 58 \\
\hline Subject-2 & 20 & 1.65 & 53 \\
\hline
\end{tabular}

Firstly, the subjects were prepared for the experiment through having a light breakfast in the morning comprising of the same food items. The measurements were taken two and a half hour later. The six electrodes (conventional disposable ECG electrodes) were placed on the abdomen so that the focused zone corresponds to the lower part of the stomach as shown in Fig.3, in which an outline of the stomach is also superimposed to show the approximate position. A reference electrode is placed on the back of the thorax, just behind the measuring electrodes, which is connected to the ground of the potential measuring circuit.

On the first day, several initial FIM measurements were taken on a subject with empty stomach, and the average of the readings were taken. The subject then had a drink of $500 \mathrm{ml}$ saline of conductivity $8 \mathrm{mS} / \mathrm{cm}$ (measured using a conductivity meter), and another quick set of FIM measurements were 
carried out immediately after the drink, and the average taken. During every measurement, the subject was asked to breathe in fully and to hold breath at that point so that the position of the stomach remains unchanged for each measurement. Breathing in also reduces the current through the adjacent lungs as these are filled with insulating air. The subject was at standing position during measurements, again for reproducibility. Similar measurements were carried out on two other days with the subject drinking saline of the same conductivity but with volumes of $250 \mathrm{ml}$ and $100 \mathrm{ml}$ respectively. The timings of breakfast and measurement, and the items for breakfast were all kept the same. The same measurements were carried out on subject- 2 on different days.

As mentioned before, since the amplitude of the driven currents was constant, the measured potentials across the two central electrodes were proportional to the total measured transfer impedance. The radius and cross sectional area of the saline in the stomach were estimated assuming the saline to occupy a spherical volume.

\section{RESULTS}

The measured transfer impedances corresponding to different volumes of saline intake for the two subjects are shown in Table 2. The first row in each shows the reference value for empty stomach and is included to facilitate normalisation and graph plotting. Normalised values of the parameters $Z_{\mathrm{s}} / Z_{\mathrm{e}}$ and $\left(Z_{\mathrm{e}}-\mathrm{Z}_{\mathrm{s}}\right)$ are also shown. In Figures 4, 5 and 6 these values are plotted against respective radii, cross sectional areas and volumes of saline in stomach, assuming the liquid to take up a spherical shape in the stomach, as mentioned before. Focusing on $\left(\mathrm{Z}_{\mathrm{e}}-\mathrm{Z}_{\mathrm{s}}\right)$ it can be seen that the variations are non-linear while it is very close to a linear shape for the third, i.e., for the plot against volume. Linear fitting for all the four plots (using trendline function of EXCEL software) are shown in Figure 7 where the fitted equations are also shown. It can be seen that the normalised $\left(Z_{\mathrm{e}}-Z_{\mathrm{s}}\right)$ plots for the two subjects almost overlap, having exactly the same slope but a very small difference in the intercept. The plots for normalised $\left(Z_{\mathrm{e}}-Z_{\mathrm{s}}\right)$ diverged to some extent.

Table-2: Measured transfer impedances and analysed parameters corresponding to different volumes of saline intake for two subjects

\begin{tabular}{|c|c|c|c|c|c|c|c|r|}
\cline { 2 - 10 } \multicolumn{1}{c|}{} & \multicolumn{4}{c|}{ Subject-1 } & \multicolumn{3}{c|}{ Subject-2 } \\
\hline $\begin{array}{c}\text { Saline } \\
\text { volume } \\
\text { in } \\
\text { stomach }\end{array}$ & $\begin{array}{c}\text { Impedance } \\
\text { with } \\
\text { empty } \\
\text { stomach } \\
\text { Ze }\end{array}$ & $\begin{array}{c}\text { Impedance } \\
\text { with } \\
\text { saline in } \\
\text { stomach } \\
\text { Zs }\end{array}$ & $\begin{array}{c}\text { Normal } \\
\text {-ised } \\
\text { Zs/Ze }\end{array}$ & $\begin{array}{c}\text { Normal } \\
\text {-ised } \\
\text { Ze-Zs) }\end{array}$ & $\begin{array}{c}\text { Impedance } \\
\text { with } \\
\text { empty } \\
\text { stomach } \\
\text { Ze }\end{array}$ & $\begin{array}{c}\text { Impedance } \\
\text { with saline } \\
\text { in } \\
\text { Stomach } \\
\text { Zs }\end{array}$ & $\begin{array}{c}\text { Normal } \\
\text {-ised } \\
\text { Zs/Ze }\end{array}$ & $\begin{array}{c}\text { Normal- } \\
\text { ised } \\
\text { (Ze-Zs) }\end{array}$ \\
\hline $\mathrm{ml}$ & $\begin{array}{c}\text { arbitrary } \\
\text { scale }\end{array}$ & $\begin{array}{c}\text { arbitrary } \\
\text { scale }\end{array}$ & & & $\begin{array}{c}\text { arbitrary } \\
\text { scale }\end{array}$ & $\begin{array}{c}\text { arbitrary } \\
\text { scale }\end{array}$ & & \\
\hline 0 & 1.288 & 1.288 & 1.000 & 0.000 & 1.5 & 1.5 & 1.000 & 0.000 \\
\hline 100 & 1.288 & 1.23 & 0.955 & 0.172 & 1.527 & 1.458 & 0.955 & 0.226 \\
\hline 250 & 1.588 & 1.394 & 0.878 & 0.576 & 1.608 & 1.436 & 0.893 & 0.564 \\
\hline 500 & 1.642 & 1.305 & 0.795 & 1.000 & 1.85 & 1.545 & 0.835 & 1.000 \\
\hline
\end{tabular}



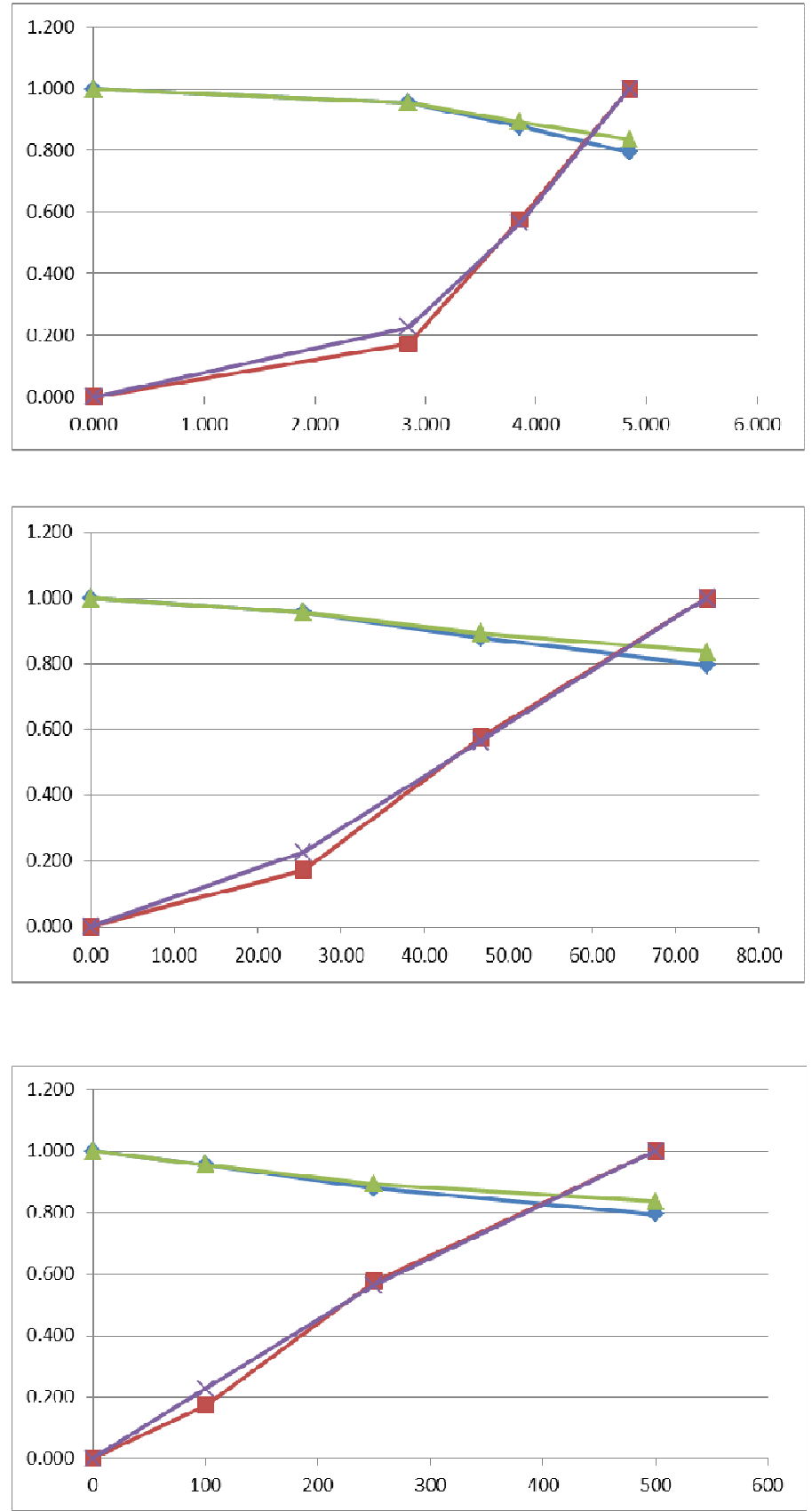

Fig. 4: Measured values plotted against radius of assumed sphere (in $\mathrm{cm}$ ) of saline in stomach for Subjects 1 and 2.

Top curves: normalised $\mathrm{Z}_{\mathrm{S}} / \mathrm{Z}_{\mathrm{e}}$

Bottom curves: normalised $\left(\mathrm{Z}_{\mathrm{e}}-\mathrm{Z}_{\mathrm{s}}\right) / \mathrm{Z}_{\mathrm{e}}$

Fig. 5: Measured values plotted against cross sectional area of assumed sphere (in sq $\mathrm{cm}$ ) of saline in stomach for Subjects 1 and 2.

Top curves: normalised $\mathrm{Z}_{\mathrm{s}} / \mathrm{Z}_{\mathrm{e}}$

Bottom curves: normalised $\left(\mathrm{Z}_{\mathrm{e}}-\mathrm{Z}_{\mathrm{s}}\right) / \mathrm{Z}_{\mathrm{e}}$

Fig. 6: Measured values plotted against against volume of saline (in cc) for subjects 1 and 2.

Top curves: normalised $\mathrm{Z}_{\mathrm{s}} / \mathrm{Z}_{\mathrm{e}}$

Bottom curves: normalised $\left(\mathrm{Z}_{\mathrm{e}}-\mathrm{Z}_{\mathrm{s}}\right) / \mathrm{Z}_{\mathrm{e}}$

\section{DISCUSSION}

The present work gives an insight into the possibility of FIM in measuring the volume of a liquid in the stomach non-invasively, using skin surface electrodes on the abdomen. In particular this measurement is directed to the measurement of the absolute volume of secreted acid in the stomach which has diagnostic applications in diarrhoeal diseases and in anaemia. The version of FIM used in this work is the 6-electrode one.

Through a simplified resistance model this work has predicted that the variation in $\left(Z_{\mathrm{e}}-Z_{\mathrm{s}}\right) / Z_{\mathrm{e}}$ with volume of liquid in stomach should be much greater than that of $Z_{s} / Z_{e}$ which has been verified through experiments on the two subjects. The simple resistance model has also predicted that the 
normalised change in measured transfer impedance $\left(Z_{e}-Z_{s}\right) / Z_{e}$ will be proportional to the volume of liquid in the stomach provided the resistivity of the liquid and the depth of stomach from the frontal skin surface is constant. Through real life measurement on two young male subjects after drinks of saline this work has verified the above prediction quite well.

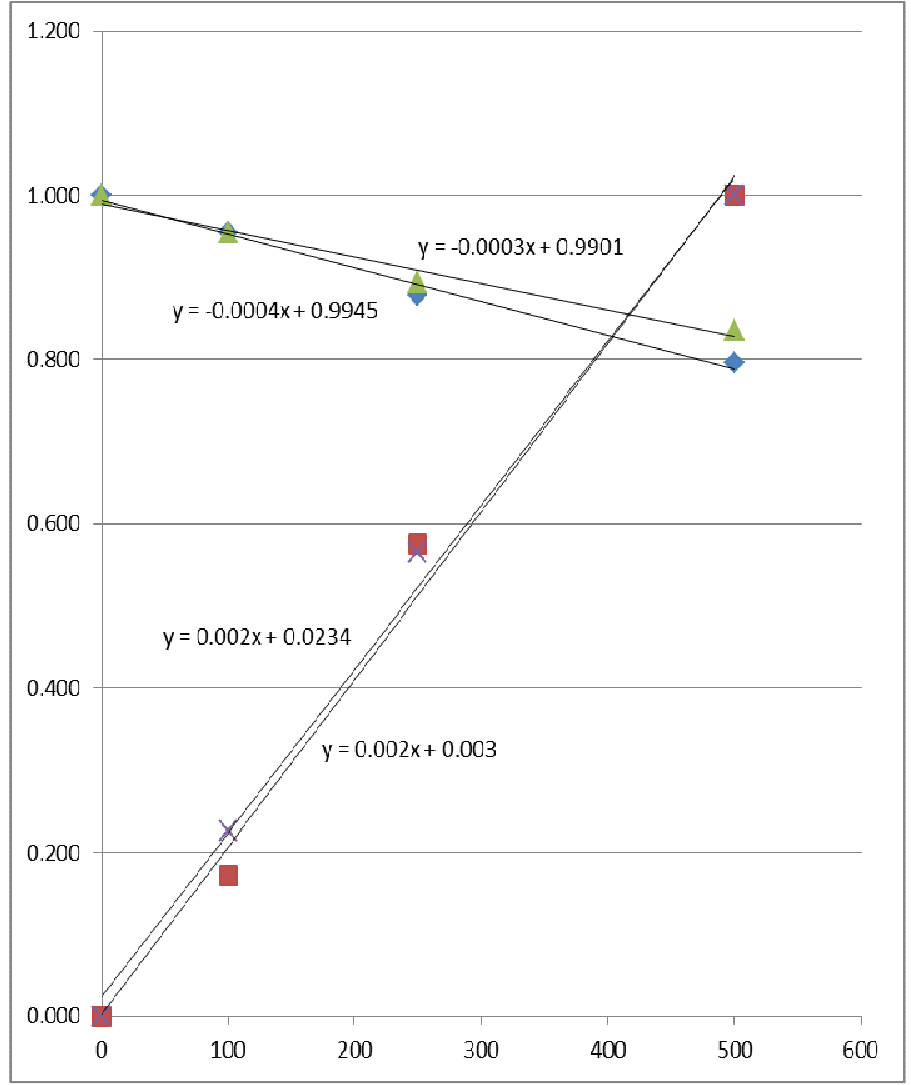

Fig. 7: Measured values plotted against against volume of saline (in cc) for subjects 1 and 2 showing fitted straight lines only.

Top curves: normalised $\mathrm{Z}_{\mathrm{s}} / \mathrm{Z}_{\mathrm{e}}$

Bottom curves: normalised $\left(\mathrm{Z}_{\mathrm{e}}-\mathrm{Z}_{\mathrm{s}}\right) / \mathrm{Z}_{\mathrm{e}}$ Note the same slope for both except for a small difference in the intercept

Both of the above findings indicate the success of the simple resistance model in analysing the volume of a target object inside a volume conductor. The good experimental agreement has increased the confidence in the method. These were carried out on the two different subjects on different days. Still the data points fitted a straight line well.

The measured behaviours were almost the same for both the subjects under test. In fact the measured values almost coincided as can be seen through the two fitted straight line plots in Fig.7 which are very close. Keeping view of experimental errors and the gross assumption of a spherical saline volume, the shapes of both of these plots support the linear fit of $\left(Z_{e}-Z_{s}\right) / Z_{e}$ against volume. This means that the measured transfer impedance change can indeed be related to the volume of saline in stomach.

It is clear that the measurement of the absolute volume of secreted acid in the stomach will need information of the depth of the stomach from the abdomen. From anatomical information of typical body frames it may be possible to estimate this thickness to a certain acceptable uncertainty. Then using information obtained for the variation of $\left(\mathrm{Z}_{\mathrm{e}}-\mathrm{Z}_{\mathrm{s}}\right) / \mathrm{Z}_{\mathrm{e}}$ with depth based on phantom measurements or numerical analyses to simulate the resistivity situation around a stomach it may be possible to determine the absolute volume. Thus the present work establishes an important step in the non- 
invasive measurement of the secreted acid volume inside the stomach, with important implications in the research and management of diarrhoeal diseases and anaemia, both prevalent in the Third World.

\section{References}

Blonski WC, Katzka DA, Lichtenstein GR \& Metz DC. 2013. Idiopathic gastric acid hypersecretion presenting as a diarrheal disorder and mimicking both Zollinger-Ellison syndrome and Crohn's disease. http://www.ncbi.nlm.nih.gov/pubmed/15756097.

Iquebal AHM \& Rabbani KS. 2010. 3D sensitivity of 6-electrode Focused Impedance Method (FIM). J. Phys.: Conf. Ser. (IOP), 224. http://iopscience.iop.org/1742-6596/224/1/012156.

Islam N, Rabbani KS \& Wilson AJ. 10th Int Con, Biomed Appl of El Imp Tomography (EIT2009) and Workshop on Electromagnetic Inverse Problems, 2009.

Islam N, Rabbani KS \& Wilson AJ. 2010. Sensitivity of focussed electrical impedance measurements. Physiol. Meas., 31. S97-S109. http://iopscience.iop.org/0967-3334/31/8/S08/.

Rabbani KS, Sarker M, Akond MHR \& Akter T. 1999. Focused Impedance Measurement (FIM) a new technique with improved zone localization. Annals of the new York Academy of Science, Electrical Bioimpedance $\quad$ Methods, $873.808-420$. http://onlinelibrary.wiley.com/doi/10.1111/j.1749-6632.1999.tb09490.x/pdf.

Sarkar Shafiqul A, Fuchs GJ, Davidsson Lena, Sattar Shamima, Alam Nur H, Mahmood Hasan \& Gyr Klaus E. 2013. Helicobacter pylori Infection: Its Role in Gastric Acid Secretion and Iron Absorption in Bangladeshi Children. http://www.icddrb.org/media-centre/factsheets/cat view/52-publications/10042-icddrb-periodicals/10073-scientificconferences/10339-10th-ascon-2002/13841-oral-session-01-nutrition-diarrhoeal-diseaseinteractions.

Sarkar Shafiqul A, Mahalanabis Dilip, Bardhan Pradip K., Alam Nur H, Rabbani KS, Kiber Adnan, Hassan Moinuddin, Islam Shafiqul, Fuchs GJ \& Gyr Klaus. 1997. Noninvasive Assessment of Gastric Acid Secretion in Man (Application of Electrical Impedance Tomography (EIT)). Digestive Diseases and Sciences (Springer Netherlands), 42. 1804-1809. http://link.springer.com/article/10.1023\%2FA\%3A1018894322528. 